\title{
Wnt2b, Wnt3, and R-Spondin-1 Promote $\beta$-Catenin Signaling in the Small Intestine during Infancy
}

\author{
Zenab M Dudhwala ${ }^{1,2^{*}}$, Rino P Donato ${ }^{1,2}$, Wendy J Uylaki ${ }^{1,2}$, Paul D Hammond ${ }^{3,4}$, \\ Gordon S Howarth ${ }^{5,6}$ and Adrian G Cummins ${ }^{1,2,3}$

${ }^{1}$ Department of Gastroenterology and Hepatology, Basil Hetzel Institute for Translational Health Research Woodville South, Australia

${ }^{2}$ The Queen Elizabeth Hospital, Woodville South, Australia

${ }^{3}$ Discipline of Medicine, School of Medicine, Faculty of Health Sciences, University of Adelaide, Australia

${ }^{4}$ School of Pediatrics, Faculty of Health Sciences, University of Adelaide, North Adelaide, Australia

${ }^{5}$ Women's and Children's Hospital, North Adelaide, Australia

${ }^{6}$ School of Animal and Veterinary Sciences, University of Adelaide, Rose worthy, Australia

\begin{abstract}
Background and aim: The Wnt- $\beta$-catenin signaling pathway is active in the stem cell region of intestinal crypts during postnatal growth of the small intestine. This functions to construct and maintain an intestinal stem cell niche in the base of crypts and to protect stem cells from apoptosis. The aim of this study was to identify which Wnt agonists are present to mediate this activity.

Methods: RNA was extracted from rats at day 14 infant $(n=4)$ and day $72(n=4)$ of life and analysed by real time PCR array to identify which Wnts were up regulated during infancy with selected Wnts localized by laser capture microscopy and PCR. Subsequently, Wnts were assessed in intestinal homogenates by individual Wnt real time quantitative PCR using different primer sets from litters $(n=6)$ at $7,14,21,28,35$ and 72 days of life in rats. Wnt3 and R-spondin-1 were also assessed in endoscopic duodenal biopsies from three infants, seven children and eight adults using immunohistochemistical staining and quantified by image analysis. Wnts were also examined by quantitative immunohistochemical and image analysis.

Results: In infant rats, seven of 18 Wnts were significantly up regulated by real time PCR array. Using laser capture microscopy, Wnt3 was exclusively present in the crypt base mainly in the epithelium of infant rats. Wnt4, Wnt5b and Wnt9b were expressed in villous epithelium and were not expressed in the crypt base of infant rats. Wnt2b expression peaked early in young infant rats ( 7 and 14 days) while Wnt3 increased 9 -fold in infant rats (14 days). R-spondin-1 peaked during infancy and had a 4-fold increase at 14 days, Wnt3 had a 5.5-fold increase in infants and R-spomdin-1 had a 1.7fold increase in children, respectively, compared with adults. R-spondin-1 expression showed a lesser 2.7-fold increase, but a similar peak in both infants and children compared to adults.

Conclusion: Wnt2b peaked early in life, at least in rats, whereas Wnt3 and R-spondin-1 peaked during infancy. These agonists facilitate Wnt- $\beta$-catenin signaling that maintains the intestinal crypt as a niche and protects stem cells from apoptosis.
\end{abstract}

\section{Keywords}

Infant, Intestinal growth, R-Spondin, Wnt

\section{Abbreviations}

ISCs: Intestinal Stem Cell(s); LCM: Laser Capture Microscopy

\section{Introduction}

The Wnt- $\beta$-catenin pathway is active in the small intestine during infancy of both rats [1] and humans [2]. This activity promotes positioning of Paneth cells at the base of crypts to form a stem cell niche [3], and protects intestinal stem cells from apoptosis such that intestinal growth may proceed during infancy [2]. Enhanced Wnt- $\beta$-catenin signaling in the small intestine is present during infancy in both rats and humans, as shown by peaks of cytoplasmic and nuclear $\beta$-catenin, and of cytoplasmic axin-2 [1,2].

There are 19 possible Wnt agonists of which Wnt3 has been identified as the only Wnt present in the epithelial crypt 
base of adult mice [1], while Wnt2b is present in pericryptal mesenchyme which surrounds the crypt in neonatal mice [2]. Besides Wnts, R-spondins-1 to 4 also acts as agonists for Wnt$\beta$-catenin signaling. Wnts exert their mechanism of action at short range by direct cell to cell apposition of secreted Wnt with frizzled-7, the cognate Wnt receptor. Wnt3 is produced by Paneth cells [3]. Wnt2b (formerly Wnt13) [4], and R-spondins -1 to -4 are produced by the mesenchymal shroud $[5,6]$. R-spondin-1 is soluble and therefore is used experimentally $[5,7]$. Typically, researchers use genetically manipulated mice, or culture medium containing R-spondin-1 to assess Wnt expression of intestinal organoids [8]. R-spondin-1 has low expression in the intestine [1], and the less soluble R-spondins- 2 and -3 , are the main R-spondins in the intestine [9]. R-spondins promote high levels of Wnt activity, as neutralizing antibodies to either R-spondin-2 or -3 inhibit tumour growth by a decrease in intestinal stem cell markers such as Lgr5 [10]. Furthermore, recovery after radiation injury is markedly impaired after neutralization of R-spondin-3 (10). R-spondin-3 may require an inflammatory response for its release as shown in dextran sulphate induced colitis [11].

Intestinal stem cells are located at the base of intestinal crypts admixed with Paneth cells $[12,13]$. The stem cell niche consists of an epithelial compartment compromising slender intestinal stem cells alternating between vacuolated Paneth cells which are surrounded by a mesenchymal pericryptal shroud. Other suggested Wnt agonists are Wnt4 and Wnt6 in the small intestine of adult mice [2]. The Wnt- $\beta$-catenin pathway is involved in organogenesis and homeostasis of a variety of adult epithelial tissues. Examples include small and large intestine, breast, pancreas and hair follicles [14]. Nineteen possible Wnt ligands engage a tripartite receptor complex of one of a potential 10 frizzled proteins, low density lipoproteins 5 or 6 , and R-spondins- 1 to -4 . Wnts engage the receptor by covalent bonding using a fatty acid residue involving Wingless and PORCN genes [15]. Activation of the Wnt receptor leads to degradation of the 'destructive complex' that maintains low $\beta$-catenin cellular levels. Axin-2 is a component of the scaffold of destructive complex that maintains low levels of $\beta$-catenin which prevent nuclear translocation of $\beta$-catenin. Wnt signalling results in phosphorylation of the destructive complex and removal by the proteosome. Phosphorylation of $\beta$-catenin occurs and cytoplasmic and nuclear levels of $\beta$-catenin increase. Activated $\beta$-catenin displaces Groucho and allows binding of the Tcf-4 transcription factor to DNA to transactivate Wnt target genes $[16,17]$.

We initially utilised infant rats and human infants and investigated Wnt $2 \mathrm{~b}$ expression only in rats, but not in humans. This was because intestinal ontogeny in rats is delayed with respect to humans. For example, crypt formation occurs during the first week of life in rats but at the start of the second trimester in humans $[18,19]$. Consequently, the aims of the current study were to investigate which Wnt agonists are present in the stem cell niche of the small intestine postnatally.

\section{Methods}

\section{Subjects}

Humans: Duodenal biopsies were collected during endoscopy from three infants and 13 children who were investigated for reflux oesophagitis or non-ulcer dyspepsia. Subjects had no macroscopic evidence of duodenal pathology. Subsequent duodenal biopsies taken for clinical purposes were reported as normal on expert pathological examination. Some of these biopsies were used in a previous study [20]. This study was approved by the Human Ethics Committee of the Women's and Children's Health Network (Adelaide) (HREC/1982/09/2022).

Rats: Litters of Hooded Wistar rats were acquired from the University of Adelaide Laboratory Animal Services (Gilles Plains, Adelaide, South Australia, Australia) and bred with day 0 designated as the day of birth. This study was approved by the joint Central Northern Adelaide Health Service/Institute of Medical and Veterinary Science Animal Ethics Committee (30-18) and the Animal Ethics Committee of the University of Adelaide (M-2016-017A).

Real time PCR array: A Wnt Pathway $\mathrm{RT}^{2}$ profiler real-time PCR array that comprised primers for 84 genes of the Wnt$\beta$-catenin pathway (including 18 of the $19 \mathrm{Wnt}$ ligands) was used (SABiosciences, Frederick, MS, USA). RNA from rats aged 14 days $(n=4)$ and 72 days $(n=4)$ of life were used according to the manufacturer's instructions. Proprietary webbased Quant studio Software V1.2.4 (Applied Biosystems, Melbourne, Victoria, Australia) was used to analyse data.

Quantitative real time PCR: Primers were designed using Genitals (Premier Biosoft International, Pato Alto, CA, USA) for candidate Wnt genes (Supplementary Table 1). Efficiency and linearity of amplification were confirmed over a 5-decade cDNA concentration range. Data were normalized to $\beta$-actin using the $2^{-\Delta \Delta C}$ method [21]. RPLO was used as a stable reference gene in the small intestine [22] which was confirmed by comparing microarray expression between infant and adult rats [23]. RNA was obtained from the small intestine of litters $(n=6)$ of rats at 7, 14, 28, 35 and 72 days of life.

Laser capture micro dissection (LCM): Cryostat sections of rat small intestine at day 14 of life were used to extract RNA from the crypt epithelium, the mesenchyme surrounding the crypt (pericryptal mesenchyme), the whole villus (epithelium

*Corresponding author: Zenab Dudhwala, Gastroenterology Research Laboratory, Basil Hetzel Institute for Translational Health Research, Australia; The Queen Elizabeth Hospital, Woodville South, South Australia 5011, Australia, Tel: +6188222-7076

Accepted: April 22, 2021

Published online: April 24, 2021

Citation: Dudhwala ZM, Donato RP, Uylaki WJ, et al. (2021) Wnt2b, Wnt3, and R-Spondin-1 Promote $\beta$-Catenin Signaling in the Small Intestine during Infancy. Insights Stem Cell Res Ther 3(1):31-39 

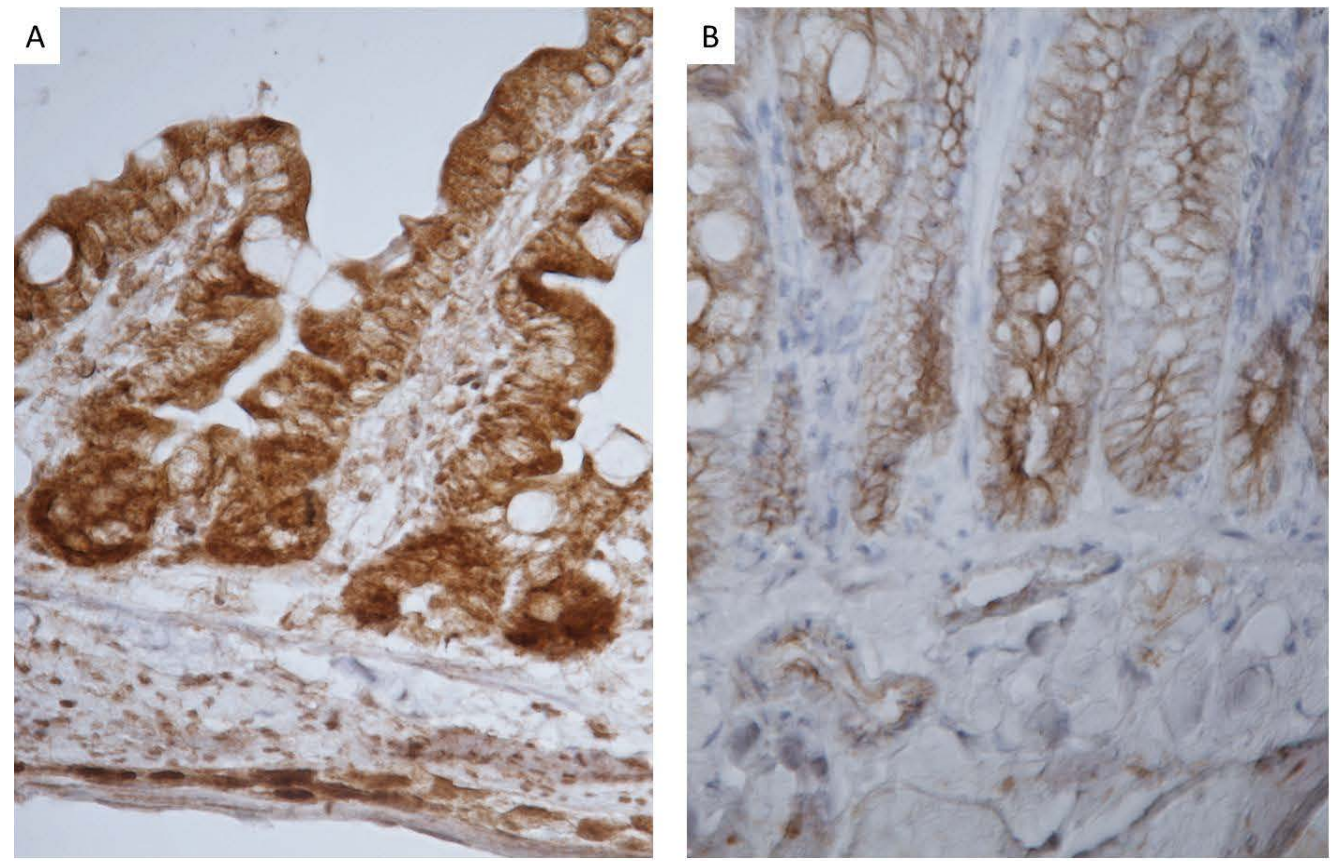

Figure 1: Representative photomicrographs of Axin-2 (A,B) expression in the intestinal crypts of day 14 (A) and 72 (B) old rats. Short bifid crypts are present in rats at 14 days of age (A). Protein expression of Axin-2 was high during infancy but was weak in adult rats. Images were captured at $\times 400$ magnification.

plus lamina propria) the villous epithelium and the villous mesenchyme utilising a Leica laser capture microscope (Leica LMD6000, Leica Microsystems, Welzlar, Germany). CDNA was made using these extracts and qRT-PCR analyses performed to compare expression of candidate Wnt ligand.

Immunostaining: Jejunal samples from rats at 14 and 72 days of life were fixed overnight in formalin and embedded in paraffin blocks. Immunostaining used an indirect polymer immunoperoxidase technique. Rabbit anti Axin-2 (1:5000 in rats, Abcam, Melbourne, Victoria, Australia, ab 109307) and anti-Wnt3 monoclonal antibodies (dilution of 1:500 in humans and 1:2000 in rats) (Abcam, Melbourne, Victoria, Australian ab 32249) were applied to the tissue and incubated overnight at $4{ }^{\circ} \mathrm{C}$. Bound antibody was detected by a secondary polymer anti-rabbit/mouse horseradish peroxidase antibody (Dako, North Sydney, New, South Wales, Australia). Bound antibody was detected by diaminobenzidine chromogen. Similarly, Wnt2b was immunostained in rats with a rabbit monoclonal antibody at 1:500 dilution (Assay Matrix Pty Ltd, Melbourne, Victoria, Australia, Orb 13761).

Immunohistochemical staining analysis: Photomicrographs (Nikon, Coherent Scientific, Pty Ltd Adelaide, SA, Australia) were taken with the camera gamma functions set to 1.00 for a linear response. Regions of interest comprising an area of $25 \times 25$ pixels were taken of specific and non-specific antibody stained intestinal crypts in serial sections. A dry $\times 60 /$ NA 0.95 Nikon objective lens (Nikon Eclipse microscope, Coherent Scientific, Adelaide, SA, Australia) was used. Experimental and control regions of interest were selected of morphologically defined (slender, sparse cytoplasm, vertical nucleus) stem cells. These regions of interest were converted to $3 \times 25 \times 25$ matrices representing red, green and blue colour space. The norm function (Mat lab,
The Math works Australia, Sydney Australia) was applied and the difference between experimental and control derived matrices expressed as energy absorbed in zero-dimension units [24,25]. This technique is independent of factors pertaining to the same slide if the experimental and control serial sections are mounted on the same slide, such as cover glass thickness, light source and counter stain $[24,25]$.

Statistical analysis: Data are presented as arithmetic mean and SD if data were normally distributed whilst PCR data are presented as geometric means after transformation by $\log _{10}(x+1)$ to stabilise the variance. Continuous linear regression in humans and categorial linear regression in rats were used to analyse data in SPSS (Statistical Products and Service Solutions, IBM, New York, USA) Individual paired comparisons were made with Turkey's F test. P-value $<0.05$ was considered significant.

\section{Results}

\section{Immunohistochemical staining}

High cytoplasmic axin-2 levels indicate Wnt- $\beta$-catenin signaling. Axin-2 peaked in humans during infancy (2) and was present in the villous and crypt epithelium in rats (Figure 1). Jejunal crypts revealed much darker staining at the base of the crypts. Staining was both nuclear and cytoplasmic for rats at day 7, 14, 21 and 28 days of life. However, over time, staining became more localized to the membrane indicating cytoplasmic staining. There were significant differences in Axin-2 staining in rats at day 14 of life compared to rats at 28 , 35 and 72 days of age, $p<0.0001$. Image analysis of Axin- 2 staining in the crypt cytoplasm peaked highest at day 14 (267 \pm 37 pixel energy) compared to (120 \pm 11 pixel energy) at day 72 of life (Figure 1). 

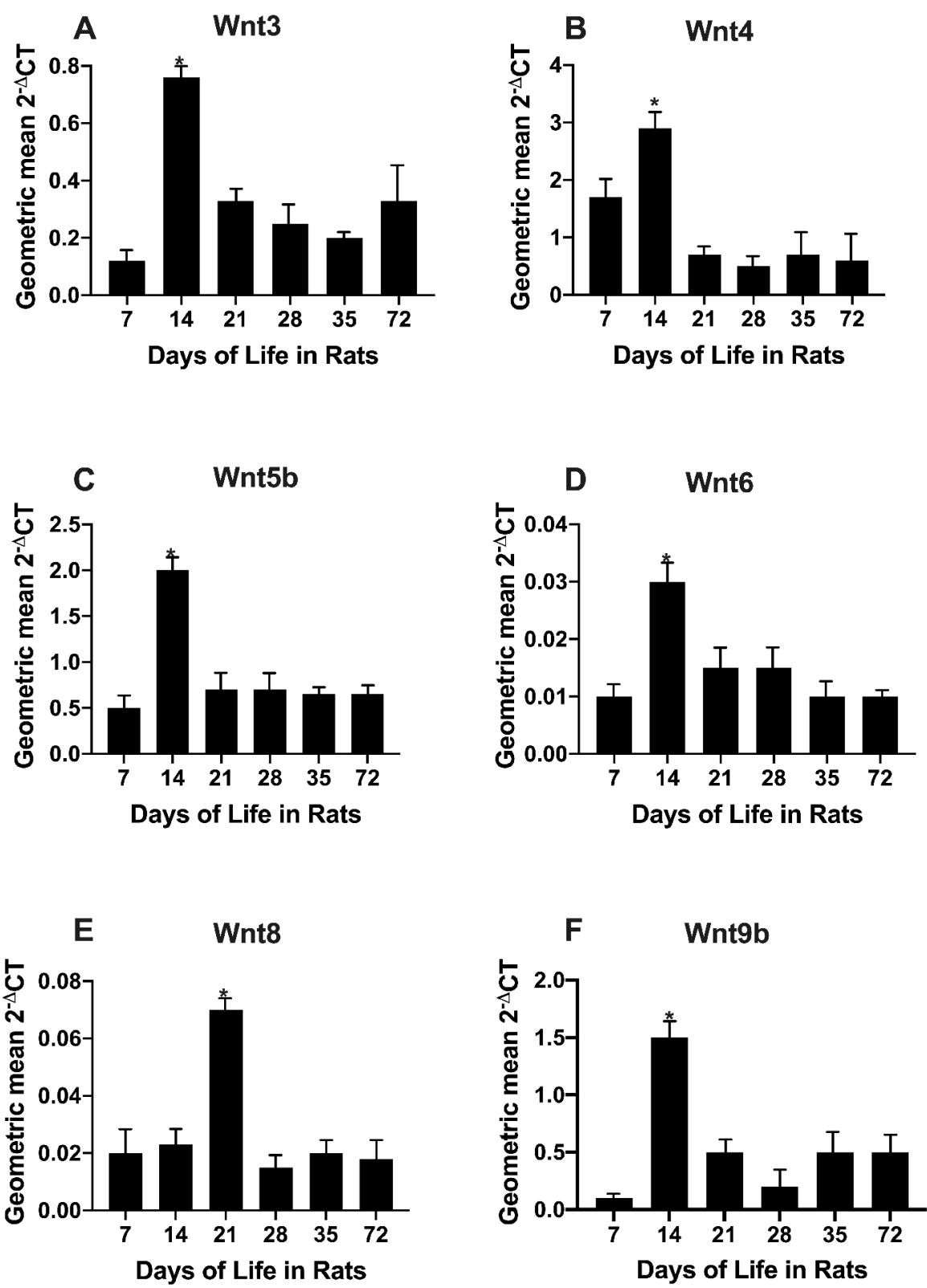

Figure 2: Age dependence of Wnt RNA expression in the jejunum of rats $(n=6)$ from 7 to 72 days of life. QRT-PCR used different primers to those used in the PCR array. Expression of Wnt3, Wnt4, Wnt5b, Wnt6 and Wnt9b were significantly elevated in rats at day 14 of life $(p<0.0001, p<0.001, p=0.03, p<0.001, p<0.001$, respectively) compared to other time points. However, Wnt8b was significantly increased at day 21 of life $(p<0.001)$ compared to any other time points. Data are expressed as mean $+S D(n=6)$ using $2^{-\Delta \Delta C}{ }_{t}$ method.

\section{Real time PCR array}

Fifty-five of 84 pathway intestinal genes from infant and adult rats were up regulated. The real time PCR array revealed 18 of the 19 possible Wnts (except Wnt16). Seven of these had a significant fold increase at day 14 of age in rats compared to day 72 (Figure 2).

\section{Age dependence of intestinal Wnts in rats from 7 to 72 days of life}

Using our own primer sets (Supplementary Table 1), we determined the age dependence of rats from 7 to 72 days of life (Figure 2). Six of the seven (Wnt3, Wnt4, Wnt5b, Wnt6,
Table 1: Comparison of intestinal levels of $18 \mathrm{Wnts}$ at 14 and 72 days of age in rats, with those having a significant fold increase displayed.

\begin{tabular}{|l|l|l|}
\hline Gene & Fold difference (14 versus $\mathbf{7 2}$ day old) & P Value \\
\hline Wnt3 & 3.91 & 0.026 \\
\hline Wnt4 & 4.58 & 0.015 \\
\hline Wnt5b & 2.99 & 0.016 \\
\hline Wnt6 & 2.09 & 0.020 \\
\hline Wnt8b & 3.34 & 0.003 \\
\hline Wnt9b & 7.14 & 0.004 \\
\hline Wnt10a & 2.67 & 0.026 \\
\hline
\end{tabular}



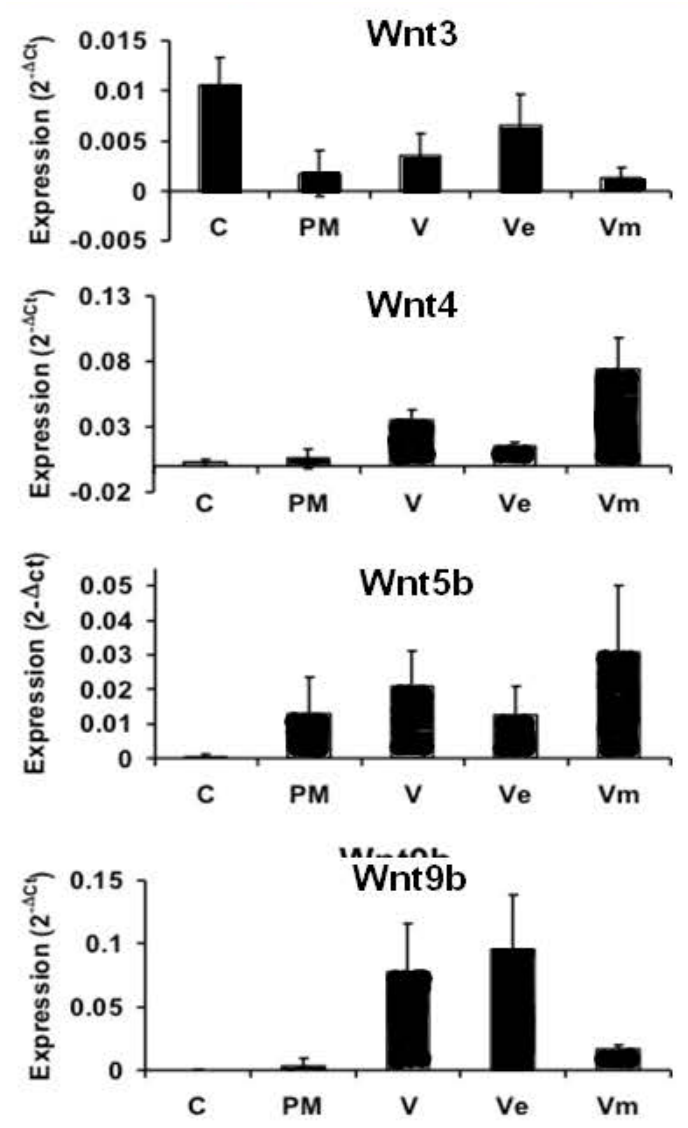

Figure 3: A) Comparison of RNA expression of Wnt3, Wnt4, Wnt5a, and Wnt9b in the crypt epithelium (C), pericryptal mesenchyme (PM), whole villus (V), villous epithelium (Ve), and villous mesenchyme (Vm). Wnt6, Wnt8b and Wnt10a were not detected. Jejunal samples were taken from four rats at 14 days of life. Expression normalized to RPLP0 and expressed in arbitrary units as mean +/- SD, the $2^{-\Delta c t} \operatorname{method}^{3}(n=4)$. B) PCR products from samples from crypts, crypt mesenchyme and villus were assessed. The absence of keratin 19 and Lgr5 products from the crypt mesenchyme extract indicates that it is free from contamination with crypt epithelial cells. However, the amplification of Lgr5 from the villous extract suggests contamination with crypt epithelial cells. The lack of MAdCAM-1 product from the crypt mesenchyme extract could be explained by the absence of venules from the captured region.

Wnt8b, Wnt9b) Wnts were initially identified by PCR array of infant rats. All Wnts except Wnt8 were confirmed as having elevated levels at 14 days of life compared to day 72 using individual qPCR.

\section{Localisation of Wnt3, Wnt4, Wnt5a, Wnt9b by LCM}

LCM was carried out on cryostat section in rats at day 14 of life. qRT-PCR analysis of Wnt ligand expression in different laser capture microdissected regions (Figure $3 \mathrm{~A}$ ) showed Wnt3 was the only Wnt to be expressed in the crypt epithelium. Keratin 19 was used as an epithelial marker, MAdCAM-1 as the marker of venule endothelium of the lamina propria and Lgr5 as intestinal stem cell marker (Figure 3B). These showed minimal cross contamination for crypt epithelial and crypt mesenchymal preparations as evident on the gel (Figure $3 \mathrm{~B})$. This confirms that Wnt3 was the only Wnt ligand mRNA amplified from the crypt epithelium, and was also amplified from the crypt mesenchyme, supporting a role for this Wnt in driving crypt fission.

\section{Age dependence of Wnt2b, Wnt3 and R-spondin-1 protein expression in rats}

Wnt2b expression was high at 7 and 14 days of life (Figure 4C). Wnt3 was assessed by immunostaining over the age range of 7 to 72 days of life (Figure 1). Wnt3 expression peaked 9-fold during infancy (14 versus 72 days) of life and decreased thereafter (Figure 4A). In contrast, R-spondin-1 had a more modest 3-fold peak in infant rats (14 days of life) versus adult rats ( 72 days of life) (Figure 4B). Thus, both Wnt3 and R-spondin-1 were available to promote activation of $\beta$-catenin during infancy in rats.

\section{Age dependence of Wnt3 and R-spondin-1 expression in human infants, children and adults}

Wnt3 expression was elevated 5 -fold in infants which decreased to 2-fold elevated in children compared to adults (Figure 5). The peak of R-spondin-1 was blunted with R-spondin-1 having a 3.9-fold elevation in both infants and children over that of adults (Figure 5).

\section{Discussion}

We initially showed that Wnt3, Wnt4, Wnt5b, Wnt6, Wnt8b, Wnt9b and Wnt10b were up regulated in the intestine of infant, compared to adult rats (Table 1). Expression of six of these 

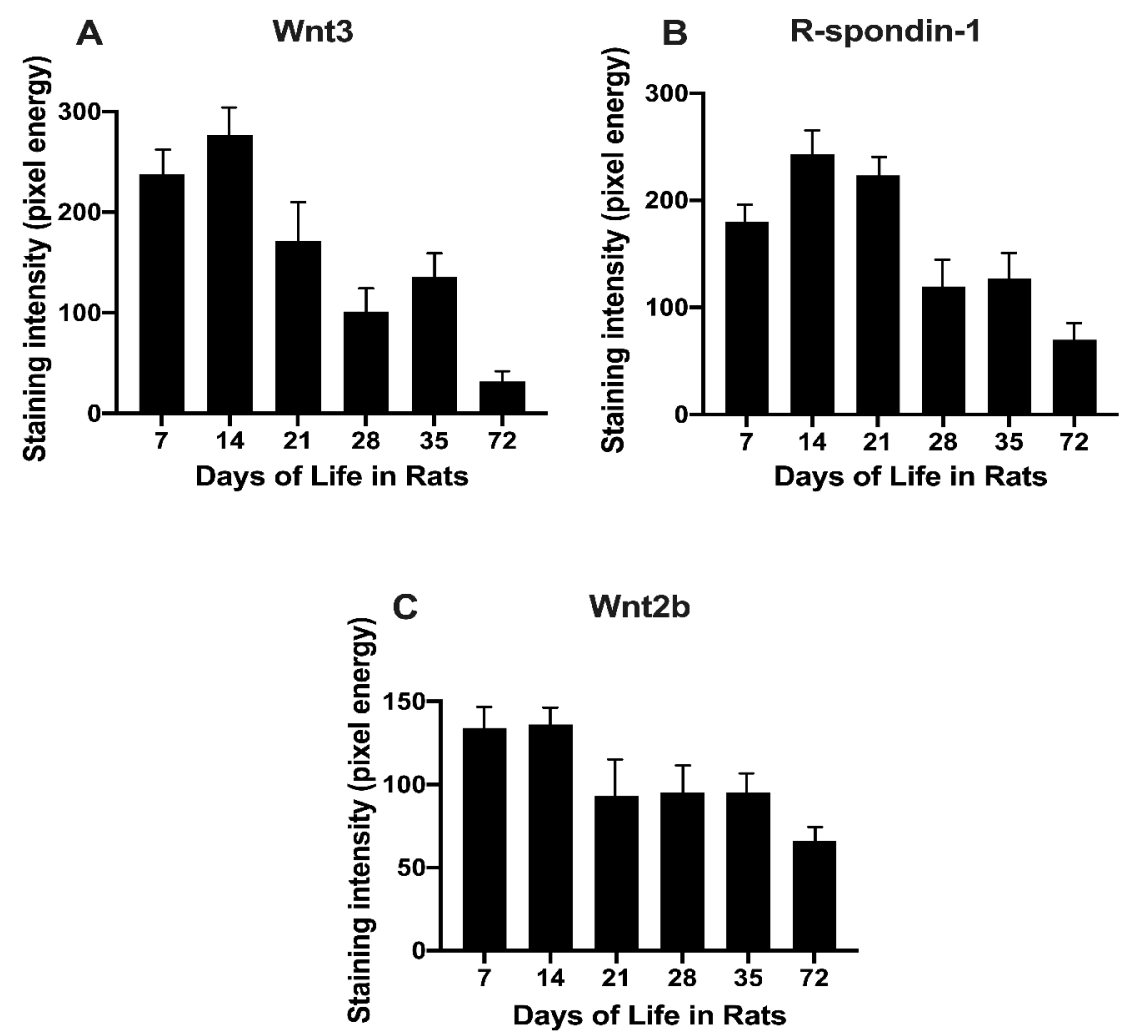

Figure 4: Protein expression of Wnt3 (A), R-spondin-1 (B) and Wnt2b (C) in the small intestine of rats after immunostaining. Staining intensity (mean + SD pixel energy) are given. Data were analysed for significance by categorical linear regression. Wnt3 staining was significantly elevated at day 14 of life in rats than compared to rats at day 28,35 and 72 of life, $p<0.0001$. Similarly, R-spondin- 1 peaked at day 14 old started to decline at day 28 of life in rats, $p<0.001$. Signal intensity for Wnt $2 b$ was significant at 14 day compared to day 72 old rats $(p=0.03)$ Wnt2b was not significant at any other time points.
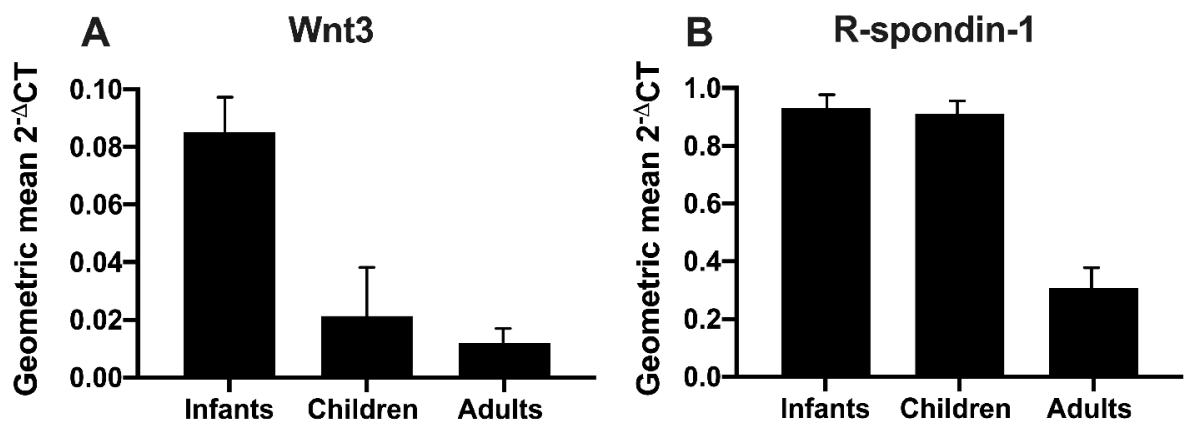

Figure 5: Wnt3 (A) and R-spondin-1 (B) expression of Wnt signaling pathway agonists by qRT-PCR from the human biopsies. Data are given as geometric mean + SD. Data were analysed for significance by categorical linear regression, $p<0.001$.

seven Wnts (except Wnt10b) peaked at 14 days of life when both intestinal crypt fission and Lgr5 stem cell expression also peak (Figure 2) $(1,2,20)$. Active $\beta$-catenin signalling promotes intestinal stem cells [26]. Localization of these Wnts was further examined after laser capture microdissection revealing that Wnt3 was exclusively localized to the epithelium of the crypt base in infant rats (Figure 3). Wnt3 had a 9-fold increase in the intestine of infant rats, and a 5-fold increase in human infants compared with that of adults. Expression in children fell to a 2-fold increase over that of adults. R-spondin-1, another Wnt agonist, also peaked to a lesser extent during infancy with a 3.9-fold increase in rats and a 4-fold increase in human infants over that of adults (Figure 1).

The importance of Wnt- $\beta$-catenin signaling has been shown by knockout of Tcf-4 transcription factor [19]. This is further supported by several studies that blocked Wnt signaling either using the Wnt antagonist, Dickkopf in transgenic mice [27], and rats [26] or by adenovirus over expression of Dickkopf [28], ablating Wingless [29], or PORCN gene products [15] that showed impaired crypt formation and reduced ISCs and crypt fission [2]. However, if Wnt3 is disrupted in the epithelium, but other Wnts are left intact in 
the pericryptal mesenchyme, there is no resulting abnormality $[17,15]$. This is due to redundancy of Wnt2b present in the mesenchyme [17]. We found that Wnt2b was expressed early in ontogeny in rats and we speculate that Wnt2b is expressed prenatally in humans.

Wnt- $\beta$-catenin signaling is essential for development and homeostasis of normal intestinal architecture. However, the mechanism of homeostasis is not well defined, although it is usually thought that Wnt- $\beta$-catenin promotes proliferation of stem cells, although there is little evidence that stem cells directly induce proliferation. Wnt3a is used in place of Wnt3, as recombinant Wnt3 is not biologically active; presumably because of a deficiency in post translational processing such as by palmitoylation. Wnt3a is a poor mitogen in vitro unless R-spondin- 1 and EGF are also present $[27,30]$. Wnt- $\beta$-catenin signalling has been shown to induce a gradient of expression of EphB2 and EphB3 receptors to guide Paneth cells down to the crypt base [3] where they form and maintain the intestinal niche [8]. Gehart and Clevers have summarised the function of Paneth cells as 'guardians of the crypt' [13]. Wnt- $\beta$-catenin signaling also protects the stem cell from undergoing apoptosis. We have shown a nadir of apoptosis in crypts at the peak of signaling in rats at 14 days of life [26], and recorded zero apoptosis in human infants up to 3-4 years of age before rising exponentially until late teenage years [20]. Blockade of notch signalling increased apoptosis in crypts in infant rats [31]. We speculate that Notch signalling is Wnt dependent. We believe these two mechanisms: First forming and maintaining the crypt; and second, of protecting the stem cell from apoptosis, underly intestinal 'homeostasis' thereby constituting the mechanism by which Wnt- $\beta$-catenin signalling causes 'growth'.

In summary, we have extended previous studies that reveal the presence of $W n t 2 b$ and $W n t 3$ in the small intestine of mice and we have identified $W n 2 b$ in the small intestine in rats. We further speculate that $W n 2 b$ is present prenatally in humans and we have also identified Wnt3 in the small intestine of human infants. Specifically, we have demonstrated that Wnt- $\beta$-catenin signaling is active, and peaks in the small intestine, during infancy in humans [2], promoted by enhanced peaks of Wnt agonists. No single Wnt agonist was found to be responsible, but both Wnt3 and R-spondin-1 were elevated. Nevertheless, Wnt3 had the greater fold increase, with R-spondin-1 exhibiting a lesser rise. R-spondin-3 is more insoluble than R-spondin- 1 , hence it has a greater role with pathological inflammation in human infants. Thus, our study extends the notion that Wnt3 is the primary Wnt3 available and present in the stem cell niche.

\section{Declarations}

\section{Ethics approval and consent for publication}

See Ethics in Methods.

\section{Availability of Data and Materials}

\section{Competing interest}

The authors declare no conflicting interests.

\section{Funding}

The study was funded by the Private Practice Fund of the Department of Gastroenterology, The Queen Elizabeth Hospital and by a PhD Scholarship awarded to ZMD by the University of Adelaide.

\section{Authors Contributions}

ZMD conducted the bench work and contributed to writing the draft; RPD conducted the laser capture analysis and associated PCR and contributed to writing and graphics, WJU contributed to PCR bench work and to writing; PDH contributed to recruiting human subjects; GSH contributed to formulation of design, supervision, and to writing the manuscript; AGC formulated the study, arranged the ethics application, supervised and contributed to writing the manuscript.

\section{Acknowledgements}

We acknowledge the assistance of Ms. Susan Wade and other staff of the Department of Histopathology, SA Pathology and The Queen Elizabeth Hospital.

\section{References}

1. Camac KS, Thompson FM, Cummins AG (2007) Activation of beta-catenin in the stem cell region of crypts during growth of the small intestine in infant rats. Dig Dis Sci 52: 1242-1246.

2. Dudhwala ZM, Drew PA, Howarth GS, et al. (2019) Active $\beta$-catenin signaling in the small intestine of humans during infancy. Dig Dis Sci 64: 77-83.

3. Batlle EH, Jeffrey T Henderson, Beghtel H, et al. (2002) Beta-cateniin and and TCF mediate cell positioning in the intestinal epitheium by controling the expression of EphBeta/ephrinBeta. Cell 111: 251-263.

4. Gregorieff A, Destrée O, Begthel H, et al. (2005) Expression pattern of Wnt signaling components in the adult intestine. Gastroenterology 129: 626-638.

5. Farin HF, van Es J, Clevers H (2012) Redundant sources of Wnt regulate intestinal stem cells and promote formation of Paneth cells. Gastroenterology 143: 1518-1529.

6. Valentia TV, Degirmenci B, Moor AE, et al. (2016) Wnt lignands secretd by subepithehlial mesenchymal cells are essential for the survvial of intestinal stem cells and gut homeostasis. Cell Rep 15: 911-918.

7. Kim KA, Zhao J, Oshima T, et al. (2006) Mitogenic influence of humnn R-sponin1 on the intestinal epithelium. Science 309: 256259.

8. Zhao J, de Vera J, Narushima S, et al. (2007) R-spondin1, a novel intestinotrophic mitogen, ameliorates experimental colitis in mice. Gastroenterology 132: 1331-1343.

9. Zhou WD, Elizabeth A, Dailey Megan J (2018) Obesity, independent of diet, drives lasting effects on intestinal epiithelial stem cell proliferation in mice. Exp Biol Med 243: 826-835.

10. Sato T, van Es JH, Snippert HJ, et al. (2011) Paneth cells provide the niche for Lgr5+ stem cells in intestinal crypts. Nature 469: 415-418.

11. Greicius G, Kabiri Z, Sigmundsson K, et al. (2018) PDGFRalpha+ pericryptal stromal cells are the critical source of Wnts and 
RSP03 for murine intestinal stem cels in vivo. Proc Natl Acad Sci USA 115: E3173-E3181.

12. Storm EE, Durinck S, de Souza e Melo F, et al. (2016) Targeting PTPRK-RSP03 colonic tumours promotes differentiation and the loss of stem-cell function. Nature 529: 97-100.

13. Harnack C, Berger H, Antanaviciute A, et al. (2019) R-spondin 3 promotes stem cell recovery and epithlial regeneration in the colon. Nature Communications 10.

14. Paneth J (1887) Ueber die secernirenden Zellen des Dünndarm-Epithels. Microscopic anatomy archive 31: 113.

15. Gehart H, Clevers K (2019) Tales from the crypt: New insights into intestinal stem cells. Nat Rev Gastroenrol Hepatol 16: 19-34.

16. Nusse R, Clevers H (2017) Wnt/beta-catenin signalling, disease and emerging therapeutic modalites. Cell 169: 985-999.

17. Kabiri Z, Grecius G, Maadn B, et al. (2014) Stroma provides an intestinal stem cell niche in het absence of epithelial Wnts. Development 141: 2206-2215.

18. Korinek V, Barker N, Moore P, et al. (1988) Depletion of epithelial stem-cell compartments in the small intestine of mice lacking Tcf-4. Nat Genet 19: 379-383.

19. Cummins AG, Thompson FM (2002) Effect of breast milk and weaning on epithelial growth of the small intestine in humans. Gut 51: 748-754.

20. Drozdowski LA, Clandinin T, Thomson AB (2010) Ontogeny, growth and development of the small intestine: Understanding pediatric gastroenterology. World J Gastroenterol 16: 787-799.

21. Dudhwala ZM, Howarth GS, Hammond PD, et al. (2020) Intestinal stem cells promote crypt tission during post natal growth ofthe small intestine. BMJ Open Gastroenterology 7: e000388.

22. Schmittgen TD, Livak JJ (2008) Analyzing real-time PCR data by the comparative C(T) method. Nat Procol 3: 1101-1119.
23. Dydensborg AB, Hrring E, Auclair J, et al. (2006) Normalising genes for quantitative RT-PCR in differentiating human intestinal epithelial cells and adenocarcinoma of the colon. Am J Physiol Gastrointestinal Liver Physiol 290: G1067-1074.

24. Cummins AG, Jones BJ, Thompson FM (2006) Postnatal epithelial growth of the small intestine in the rat occurs by both crypt fission and crypt hyperplasia. Dig Dis Sci 51: 718-723.

25. Matkowskyj KA, Cox R, Jensen RT, et al. (2003) Quantitative immunohistochemistry by measuring cumulative signal strength accurately measures receptor number. The Journal of Histochemistry and Cytochemistry 51: 205-214.

26. Matkowskyj KA, Schonfeld D, Benya RV (2000) Quantitative immunohistochemistry by measuring cumulative signal strength using commercially available software photoshop and matlab. J Histochem Cytochem 48: 303-312.

27. Fauser JK, Donato RP, Woenig JA, et al. (2012) Wnt blockade with dickkopf reduces intestinal crypt fission and intestinal growth in infant rats. Journal of Pediatric Gastroenterology and Nutrition 55: 26-31.

28. Pinto D, Gregotref A, Begthel H, et al. (2003) Canonical Wnt signals are essential for homeostasis if the intestinal epithelium. Genes Dev 17: 1709-1713.

29. Kubnert F, Davis CR, Wang HT, et al. (2004) Essential requirement for Wnt signaling in proliferation of adult small intestine and colon revealed by adenoviral expression of Dickkopf-1. Proc Natl Acad Sci U S A 101: 266-271.

30. Guezguez A, Paré F, Benoit YD, et al. (2014) Modulation of stemness in a human normal intestinal epithelial crypt cell line by activaiton of the Wnt signaling pathway. Exp Cell Res 322: 355-364.

31. Cummins AG, Woenig JA, Donato RP, et al. (2013) Notch signaling promotes intestinal crypt fission in the infant rat. Dig Dis and Sci 58: 678-685. 
Citation: Dudhwala ZM, Donato RP, Uylaki WJ, et al. (2021) Wnt2b, Wnt3, and R-Spondin-1 Promote $\beta$-Catenin Signaling in the Small Intestine during Infancy. Insights Stem Cell Res Ther 3(1):31-39

Supplementary Table 1: Primers used in age dependence study of rats and after laser capture microdissection of intestinal crypt base.

\begin{tabular}{|l|l|l|}
\hline Primers & $\mathbf{3}$ to $\mathbf{5}$ forward & Reverse \\
\hline B-Actin (Rat) & TGACGGTCAGGTCATCACTATCG & TTTCATGGATGCCACAGGATTC \\
\hline RPLPO (Human) & GCCCGAGAAGACTCTTTCTTCC & TGTCTCCAGTCTTTATCAGCTGCAC \\
\hline R-Spondin-1 (Human) & GCCAAAAGGTCCAGCTGCTG & CTCGGGCCCGAGACCAGTG \\
\hline Wnt3 (Human) & GTCCTCGACAGCCACCCGC & ACAGCCCAAATGGTGGAGGTG \\
\hline Wnt3 (Rat) & TCTGCTCTGCGGCTCTATCC & CTTCACGCCTTCCGCTACAC \\
\hline Wnt4 & CTGTCTTCGGGAAGGTGGG & TGTCACTGCAAAGGCCACAC \\
\hline Wnt5 & CCGAGCCCTCATGAACTTACAG & CGTGACATTTGCAGGAGACATC \\
\hline Wnt6 & GCATCCTGCAACAGGACATTC & CATAGAACAGGCCTGCGTGAC \\
\hline Wnt8b & AAGGAGAAGTACCACGCAGCAC & GTAGAGATGGAGCGAAAGGTG \\
\hline Wnt9b & CAAGGATCTGCGGGCGAGAG & TTGTTCTCAGGCCGCTCTTC \\
\hline
\end{tabular}

DOI: $10.36959 / 498 / 610$ 